\title{
Letter
}

\section{Intercalated vs Non-Intercalated Morphologies in Donor- Acceptor Bulk Heterojunction Solar Cells: PBTTT:Fullerene Charge Generation and Recombination Revisited}

\author{
Elisa Collado Fregoso, Samantha N. Hood, Safa Shoaee, Bob C. Schroeder,
} Iain McCulloch, Ivan Kassal, Dieter Neher, and James R. Durrant

J. Phys. Chem. Lett., Just Accepted Manuscript • DOI: 10.1021/acs.jpclett.7b01571 • Publication Date (Web): 04 Aug 2017

Downloaded from http://pubs.acs.org on August 8, 2017

\section{Just Accepted}

"Just Accepted" manuscripts have been peer-reviewed and accepted for publication. They are posted online prior to technical editing, formatting for publication and author proofing. The American Chemical Society provides "Just Accepted" as a free service to the research community to expedite the dissemination of scientific material as soon as possible after acceptance. "Just Accepted" manuscripts appear in full in PDF format accompanied by an HTML abstract. "Just Accepted" manuscripts have been fully peer reviewed, but should not be considered the official version of record. They are accessible to all readers and citable by the Digital Object Identifier (DOI®). "Just Accepted" is an optional service offered to authors. Therefore, the "Just Accepted" Web site may not include all articles that will be published in the journal. After a manuscript is technically edited and formatted, it will be removed from the "Just Accepted" Web site and published as an ASAP article. Note that technical editing may introduce minor changes to the manuscript text and/or graphics which could affect content, and all legal disclaimers and ethical guidelines that apply to the journal pertain. ACS cannot be held responsible for errors or consequences arising from the use of information contained in these "Just Accepted" manuscripts. 


\title{
Intercalated Vs Non-intercalated Morphologies in
}

\author{
Donor-acceptor Bulk Heterojunction Solar Cells:
}

\section{PBTTT:fullerene Charge Generation and}

\section{Recombination Revisited}

\author{
Elisa Collado-Fregoso ${ }^{1,4}$, Samantha N. Hood ${ }^{2}$,Safa Shoaee ${ }^{1}$, Bob C. Schroeder ${ }^{3,4}$, Iain \\ McCulloch ${ }^{4,5}$, Ivan Kassal ${ }^{2,6}$, Dieter Neher ${ }^{* *}$ and James R. Durrant ${ }^{4,7^{*}}$ \\ ${ }^{1}$ Department of Physics and Astronomy, University of Potsdam, Karl-Liebknecht-Straße 24- \\ 25,14476 Potsdam-Golm, Germany
}

${ }^{2}$ Centre for Engineered Quantum Systems, School of Mathematics and Physics, The University of Queensland, QLD 4072, Australia.

${ }^{3}$ Materials Research Institute and School of Biological and Chemical Sciences, Queen Mary University of London, Mile End Road, London E1 4NS, United Kingdom

${ }^{4}$ Centre for Plastic Electronics, Department of Chemistry, Imperial College London, Exhibition Road, London SW7 2AZ, United Kingdom

${ }^{5}$ KSC, King Abdullah University of Science and Technology, Thuwal 23955-6900, Saudi Arabia

${ }^{6}$ Centre for Engineered Quantum Systems, Australian Institute for Nanoscale Science and Technology, and School of Chemistry, The University of Sydney, NSW 2006, Australia ${ }^{7}$ SPECIFIC IKC, College of Engineering, Swansea University, SA12 7AX, U.K. 


\title{
*neher@uni-potsdam.de, *j.durrant@ic.ac.uk
}

\begin{abstract}
In this contribution, we study the role of the donor:acceptor interface nanostructure upon charge separation and recombination in organic photovoltaic devices and blend films, using mixtures of PBTTT and two different fullerene derivatives $\left(\mathrm{PC}_{70} \mathrm{BM}\right.$ and ICTA) as models for intercalated and non-intercalated morphologies, respectively. Thermodynamic simulations show that while the completely intercalated system exhibits a large free-energy barrier for charge separation, this barrier is significantly lower in the non-intercalated system, and almost vanishes when energetic disorder is included in the model. Despite these differences, both fs-resolved transient absorption spectroscopy (TAS) and TDCF exhibit extensive first-order losses in that system, suggesting that geminate pairs are the primary product of photoexcitation. In contrast, the system that comprises a combination of fully intercalated polymer:fullerene areas and fullerene aggregated domains (1:4 PBTTT:PC ${ }_{70} \mathrm{BM}$ ), is the only one that shows slow, second-order recombination of free charges, resulting in devices with an overall higher short circuit current and fill factor. This study therefore provides a novel consideration of the role of the interfacial nanostructure and the nature of bound charges, and their impact upon charge generation and recombination.
\end{abstract}

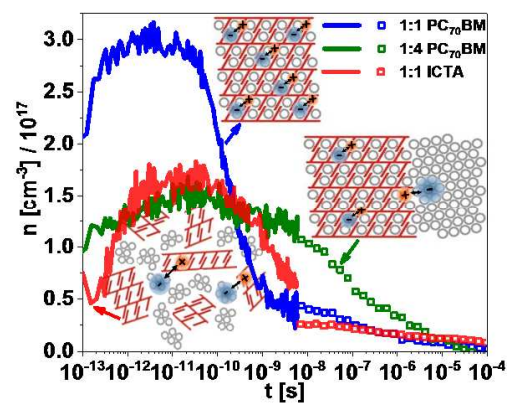


The charge separation process in organic photovoltaic blends has long been studied, however significant discrepancies remain on proposed mechanisms of free polaron generation. ${ }^{1-3}$ Variables often cited as influencing the yield of free charges are the driving energy for charge separation, ${ }^{4,5}$ the availability of excited states which support a large electron delocalisation, ${ }^{6-9}$ the dielectric constant of the blend ${ }^{10}$ and the mobility of the photogenerated charges. ${ }^{11}$ All of these factors are related to the micro and nanostructure of the blend, namely, the crystallinity, domain size and purity of the blend components. ${ }^{12-18}$ More recently, the orientation of the donor and acceptor molecules with respect to each other has been proposed as a critical nanostructure parameter, ${ }^{18,19}$ and the polarization energy at the interface has been suggested to affect the charge separation energy barrier. $^{20}$

It is generally accepted that a trade-off exists between the optimal microstructure for efficient exciton dissociation and that for efficient separation of the charges and their subsequent collection. $^{21}$ While molecularly intermixed regions of donor and acceptor are thought to be optimal for exciton dissociation, more pure, extended regions of either component have proven necessary for an efficient charge separation and collection. ${ }^{13,21-25}$ In addition, the nature of the morphological distribution in the donor-acceptor interface, whether blurred with a continuous composition gradient or presenting an abrupt transition between the donor and acceptor phase, was predicted to affect the efficiency of exciton harvesting and charge extraction, thus impacting upon the overall device performance. ${ }^{23,26,27}$ A key uncertainty in such analyses is the nature of the interfacial bound charges in such bulk heterojunction (BHJ) devices and their impact upon charge generation and recombination.

In this paper, we provide a combined experimental and theoretical study of the role of the interface nanostructure upon charge separation and recombination energetics and kinetics. We 
show, for the first time, that charge recombination kinetics at times that cover ten orders of magnitude, obtained from all-optical (fs-TAS and PL in blends) and electro-optical techniques (TDCF in devices) agree with each other in systems with efficient charge separation. Our study employs the prototypical polymer PBTTT (Poly[2,5-bis(3-tetradecylthiophen-2-yl)thieno[3,2b]thiophene] blended with different loadings of $\mathrm{PC}_{70} \mathrm{BM}([6,6]-P h e n y l$ C71 butyric acid methyl ester) and with a bulky acceptor, ICTA (indene-C60 trisadduct, see the chemical structures and UV-vis of the neat and blend films used herein in Figure S1). The critical difference between these blends is that they give rise to very particular interfacial nanostructures. ${ }^{28-31}$ The $1: 1$ PBTTT:PC ${ }_{70} \mathrm{BM}$ blend comprises fully intermixed polymer:fullerene co-crystals with a molecular interface, this system will be referred here as 'intercalated'. The 1:1 PBTTT:ICTA blend does not show intercalation, and has an abrupt interface, this system will be referred to as 'non-intercalated' and finally the 1:4 PBTTT:PC ${ }_{70} \mathrm{BM}$ blend presents both intercalated polymer:fullerene co-crystals and pure fullerene phases, and herein will be referred to as 'composite'. ${ }^{28-31}$

We find from our simulations that the free-energy of charge separation monotonically increases with increasing electron-hole distance when the PBTTT:PC ${ }_{70} \mathrm{BM}$ co-crystal is the predominant microstructure, meaning that photogenerated charges are unlikely to spontaneously separate at room temperature. However, this barrier is largely reduced for a non-intercalated sharp donoracceptor interface such as the one present in PBTTT:ICTA, and almost vanishes if energetic disorder is included in the model. In agreement with this theory, time-delayed collection field (TDCF) studies reveal a pronounced electric field dependence of charge generation in the PBTTT:PC ${ }_{70} \mathrm{BM}$ co-crystal but not in the phase-separated PBTTT:ICTA blend. Surprisingly, photovoltaic devices fabricated with the latter blend perform worst among all systems studied. 
Our transient absorption spectroscopy (TAS) and TDCF recombination studies suggest that the non-intercalated blend, suffers from delayed first-order recombination, which is consistent with the trapped charges in small and isolated PBTTT and/or ICTA domains. Importantly, geminate recombination is avoided in the composite system, the only system which comprises a combination of intercalated and pure fullerene phases. Therefore, our study highlights the importance of a multiphase morphology when aiming for efficient generation and extraction of charge in BHJ solar cells.

First, we confirm the nanostructure of the active blends. Figure 1 shows the wide angle X-Ray diffraction (WAXD) results in films of neat PBTTT and blends of PBTTT:fullerene, where it can be observed that rather large crystallites are formed in both the neat and blend films, ranging between 7 and $13 \mathrm{~nm}$ (see Table S1 for details on crystallite sizes). In the 1:1 and 1:4 PBTTT:PC ${ }_{70} \mathrm{BM}$, the lamellar peaks shift to $q=0.217 \AA^{-1}$ and $q=0.218 \AA^{-1}$ corresponding to lamellar spacings of $29.0 \AA$ and $28.8 \AA$ respectively, compared to the $20.6 \AA$ spacing in the neat PBTTT film fabricated with the same conditions. Conversely, for the non-intercalated systems, the peak remains at $q=0.309 \AA^{-1}$ corresponding to a lamellar spacing of $20.3 \AA$, very close to the lamellar spacing in the neat PBTTT film. This confirms, as previously reported, that the system which contain a sufficient amount of a small fullerene, such as the 1:1 blend with $\mathrm{PC}_{70} \mathrm{BM}$, shows predominant intercalation. ${ }^{28-31}$ This blend thus constitutes an ideal model system for a blend with only molecular heterojunctions, where every donor molecule neighbours an acceptor and vice versa. In contrast, the ICTA blend does not intercalate and therefore constitutes a model of an abrupt interface between pure phases, which we denote as a domain heterojunction. We have further confirmed these results using Resonant Raman spectroscopy. ${ }^{32}$ 


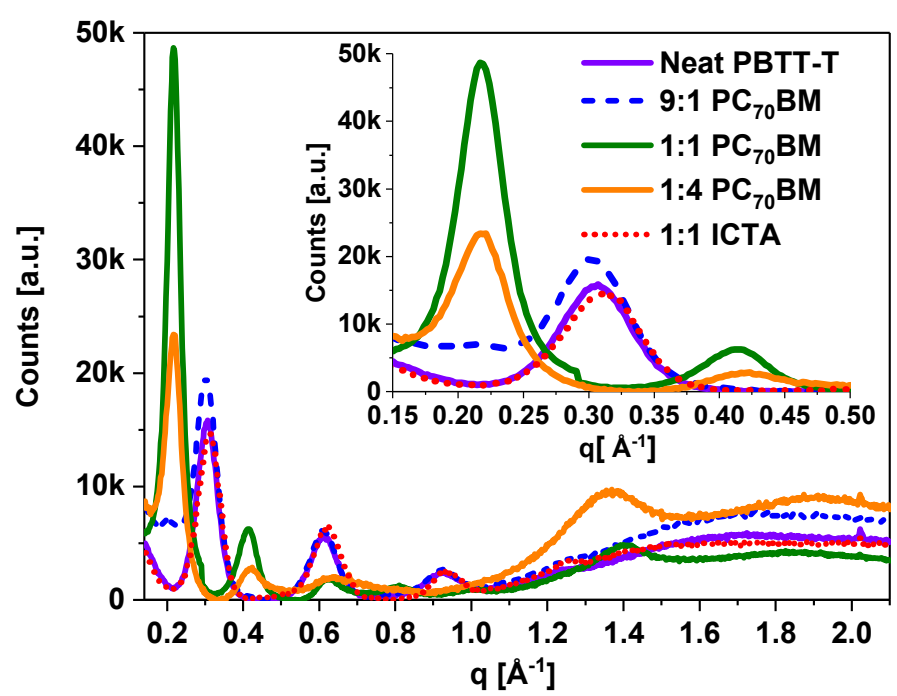

Figure 1. Wide-angle X-ray diffraction of neat PBTTT and PBTTT:fullerene thin films with different fullerene loadings.

In order to assess how the nature of the heterojunction affects the efficiency of charge generation, we performed simulations of the free energy $\Delta G(r)$ of the electron-hole pair. As we have previously described, ${ }^{33}$ the electron and hole are initially simulated as a charge-transfer (CT) state with the hole in the PBTTT and the electron in the acceptor ( $\mathrm{PC}_{70} \mathrm{BM}$ or ICTA). ${ }^{33-35}$ The free energy $\Delta G(r)$ of the electron-hole pair as a function of their separation $r$ is then calculated with and without energetic disorder. For the intercalated case, the PBTTT:PC ${ }_{70} \mathrm{BM}$ mixture is modeled as one phase with both the donor and acceptor forming one-dimensional channels, according to previous literature reports. ${ }^{30,36}$ For the non-intercalated case, the morphology was represented as two phases of three-dimensional hexagonal close-packed lattice with a planar interface between them. Additional simulation details are described in Section 2 of the SI, including Figures S2 and S3, which show a schematic representation of the two cases. 
Figure 2 shows the results of these simulations. In the intercalated case neither entropy nor disorder have a significant effect on the free energy of dissociation. In particular, there is no entropic driving because the number of accessible states does not increase with electron-hole separation when both carriers are confined to the one-dimensional channels formed in the intercalated blend. ${ }^{35}$ Conversely, for the non-intercalated case, entropy and disorder decisively lower the energetic barrier for charge-pair separation. The entropic driving comes from the fact that the number of ways of arranging two charges goes as the cube of their separation, ${ }^{33}$ lowering the free energy. Energetic disorder also lowers the barrier by allowing charges on higher-energy sites to lower their energy by moving onto neighbouring lower-lying sites, a process that is more likely to occur in higher-dimensional systems where each site has more neighbours. In particular, we notice that using $70 \mathrm{meV}$ of energetic disorder, a value estimated via Photo-CELIV for another non-intercalated system, PBTTT:bis- $\mathrm{PC}_{60} \mathrm{BM},{ }^{37}$ is enough to lower the charge separation barrier to $\approx 50 \mathrm{meV}$. This is a much lower energetic barrier compared to the barrier of more than $250 \mathrm{meV}$ calculated for the intercalated case, independent of the presence of energetic disorder. This would suggest that charge separation is energetically more favourable in a phase separated system with a more abrupt interface. We emphasize, however, that even with a small energetic barrier, charge separation need not be $100 \%$ efficient, since it is governed by kinetic properties that cannot be predicted from thermodynamic considerations; in particular, recombination could still be fast in phase separated blends, leading to low efficiencies. 


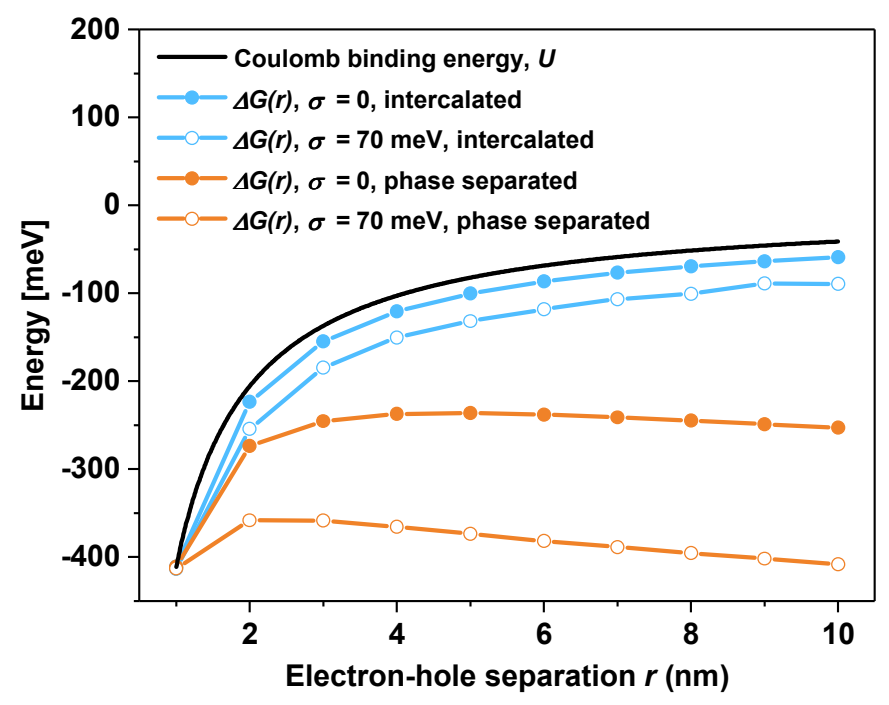

Figure 2. Electron-hole Coulombic binding (black curve) and free energy calculated without ( $\sigma$ $=0$ ) and with $70 \mathrm{meV}$ of a Gaussian energetic disorder for a completely intercalated morphology (full circles) and for a phase separated system (empty circles).

To determine whether the predominance of non-intercalated blends results in superior device performance, charge generation and recombination were studied in detail with a combination of different steady-state and transient methods. Figure 3 shows the $J-V$ curves of the PBTTT:fullerene devices (see the device parameters in Table S5) along with the external generation efficiency (EGE) obtained from our TDCF measurements, described elsewhere. ${ }^{38,39}$ Briefly, a $3.8 \mathrm{~ns}$ monochromatic (here $532 \mathrm{~nm}$ ) light pulse was shined into the device pixel while the device is held at a certain bias ('pre-bias' $V_{\text {pre }}$ ); after a certain delay time, the charges are extracted using a strong reverse bias ('collection bias' $V_{\text {coll }}$ ). EGE is calculated as the ratio of total charge carrier collected per photons shined. Data in Figure 3 is obtained by keeping the delay constant at the earliest time (6 ns) and lowest measurable intensity, $\left(0.2 \mu \mathrm{Jcm}^{2}\right)$ while the pre-bias is changed, using a collection bias $V_{\text {coll }}=-2.5 \mathrm{~V}$. 
As can be observed, the $J-V$ curves are well described (both in shape and absolute current) by our estimated EGE at reverse bias, where non-geminate charge recombination is reduced by the effect of the applied field. This means that the device photocurrent is generated exactly by the charges measured by TDCF at short delay times. It is also evident that whereas for the intercalated device the effect of pre-bias on generation is relatively large (EGE decreases by $45 \%$ from -1.5 to $0.6 \mathrm{~V})$ this effect is much less pronounced for the composite device (23\%) and slightly less for the non-intercalated device $(20 \%)$. The trend for the extreme cases i.e., intercalated vs non-intercalated, is in agreement with conclusions from earlier TDCF measurements on a similar system. ${ }^{40}$ Consistent with our simulations, this finding suggests that free charge photogeneration in the intercalated 1:1 $\mathrm{PBTTT}: \mathrm{PC}_{70} \mathrm{BM}$ device proceeds via coulombically bound intermediates -namely, charge pairs- that are bias-susceptible and 
extractable under strong applied bias. Conversely, the presence of relatively pure phases in the non-intercalated and composite devices results in charge separation significantly less dependent on the electric field - indicating a lower barrier for free charge formation in both systems. This is due to the formation of less bound charges or the formation bound charges that are less biassusceptible. Later on, we will see that the last explanation seems to fit well with the evidence obtained by other measurements for the non-intercalated, PBTTT:ICTA device.

Despite the low bias influence on EGE for the non-intercalated device, it is striking how low the absolute EGE is even at $-1.5 \mathrm{~V}$ reverse bias and how poorly this device performs, given the thermodynamic prediction of supressed geminate recombination at an abrupt domain heterojunction interface. To understand this apparent disagreement, we studied charge recombination dynamics, employing both fs-TAS and TDCF as a function of delay time. Although there have been several spectroscopic studies of PBTTT systems varying the amount of intermixed regions, ${ }^{22,31,41-45}$ a detailed analysis of charge kinetics in both blends and working solar devices where the interfacial nanostructure is controlled has been lacking.

Figure S4 shows the fs-TAS spectra and kinetics of a neat PBTTT film and a 9:1 PBTTT:PC ${ }_{70} \mathrm{BM}$ blend film. From these data, the singlet exciton photoinduced absorption band, with a maximum at $\approx 1250 \mathrm{~nm}$ can be identified. ${ }^{22}$ Its lifetime is estimated (at low intensity) to be $\tau=146 \pm 20 \mathrm{ps}$, consistent with the lifetime of other conjugated polymers used in organic photovoltaics. ${ }^{46}$ The band that appears from $\approx 850$ to $1050 \mathrm{~nm}$ for the $9: 1$ blend can be assigned to the photoinduced absorption of bound/free positive polarons in the polymer phase, consistent with other reports. ${ }^{22,42,44,45}$ These are the basis of the assignments that will be used for the rest of the blends herein presented. 
We now turn to Figure 4. Here we show, on the left column the transient spectra of the blend films. On the middle column the normalized TAS kinetics at the maximum of the bound/free polaron pairs $\left(\approx 1000 \mathrm{~nm}\right.$ for the $\mathrm{PC}_{70} \mathrm{BM}$ blends and $930 \mathrm{~nm}$ for the ICTA blend $)$ and on the right one the corresponding normalized kinetics of total charge density obtained by TDCF at $V_{\text {pre }}=0.4 V$ (near $V_{O C}$ ). As is evident in panels a and $\mathrm{d}$ for the 1:1 and 1:4 PBTTT:PC ${ }_{70} \mathrm{BM}$ blends, the exciton photoinduced absorption band is scarcely present, indicating that exciton dissociation occurs on ultrafast times, $\left(\leq 200 \mathrm{fs}\right.$ ) in agreement with previous studies, ${ }^{22,42,44,45}$ and consistent with the presence of completely intercalated areas. We note however, that this is not the case for the ICTA blend, where the exciton band is present, (see panel g) consistent with the idea that this blend contains relatively small but pure polymer and fullerene domains, where excitons have to diffuse over a certain distance in order to become separated at the donor:acceptor interface (panel h). Figure S5 shows in panel c the exciton decay kinetics of the PBTTT:ICTA blend, from where an average lifetime of $\tau=2.0 \pm 0.4$ ps for exciton dissociation was obtained from a tri-exponential fit (details of the fit are shown in Table S4). This suggests that despite the inability of ICTA to intercalate and form a co-crystal with PBTTT, the size of the pure polymer domains in its blend is fairly small. In agreement with this interpretation, the polaron photoinduced absorption shows a rise on a similar time scale, which corresponds to a fraction of polaron pairs that are generated after exciton diffusion from pure polymer areas to the interface, (see Figure 4h) also consistent with the steady-state PL measurements (Figure S5). This rise is noticeably slower than that for the intercalated system, thus emphasizing the morphological differences of the blends (see Section 4 in the SI). 

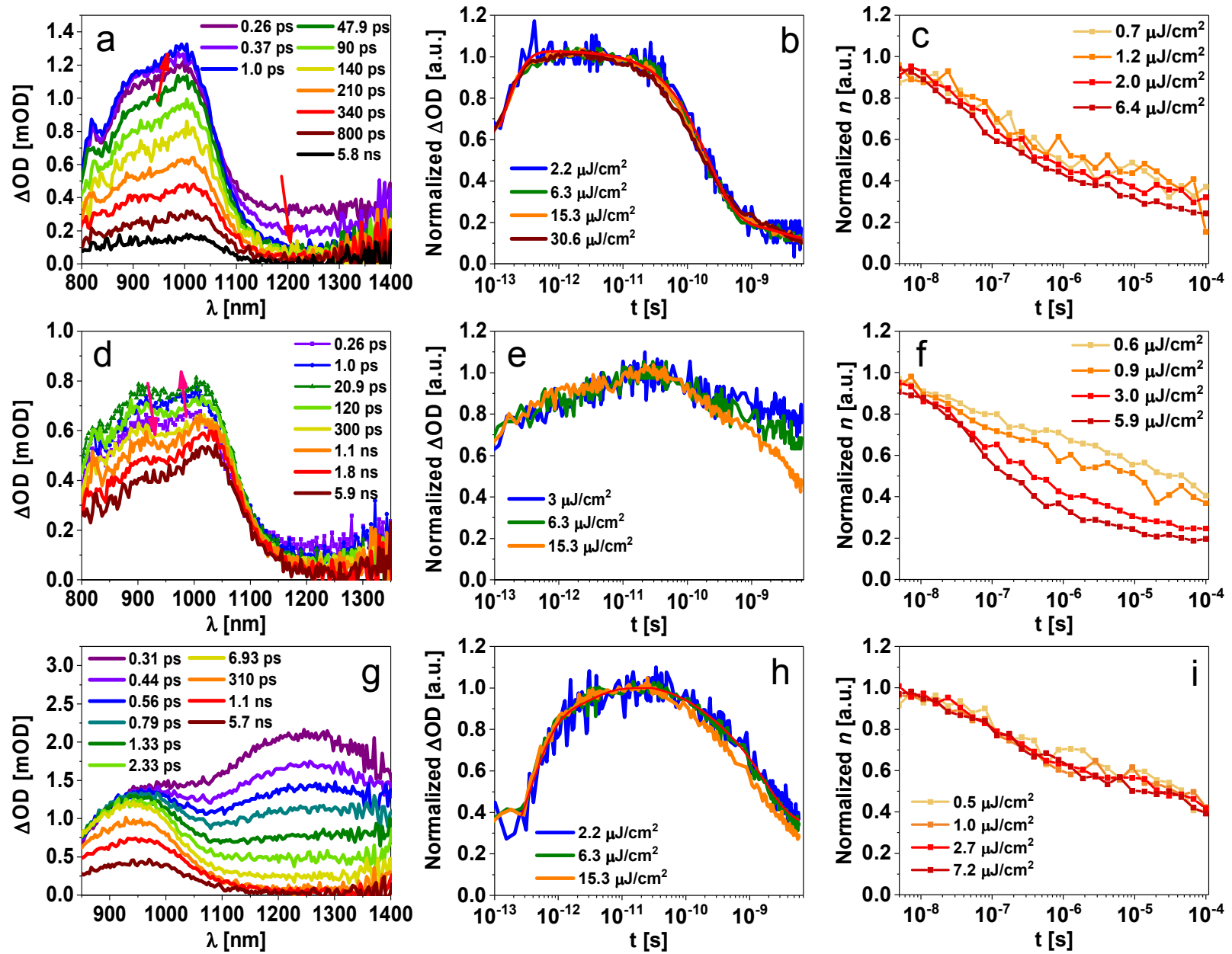

Figure 4. The three studied systems, one in each row: (a, b, c) 1:1 PBTTT:PC ${ }_{70} \mathrm{BM}$ (intercalated), (d, e, f) 1:4 PBTTT:PC ${ }_{70} \mathrm{BM}$ (composite) and (g, h, i) 1:1 PBTTT:ICTA (nonintercalated). Columns show: (a, d, g) plots of transient absorption (TAS) spectra exciting at 540 $\mathrm{nm}$ with $6 \mu \mathrm{J} / \mathrm{cm}^{2},(\mathrm{~b}, \mathrm{e}, \mathrm{h})$ normalized polaron recombination kinetics from TAS at the polaron absorption maximum, at different excitation intensities; and (c, f, i) normalized charge density from time-delayed collection field (TDCF) measurements at $0.4 \mathrm{~V}$ prebias and different light pulse intensities. Red lines in plot $\mathrm{b}$ and $\mathrm{h}$ correspond to tri-exponential fits of the data. Plot $\mathrm{g}$ ) shows the raw TAS spectra before subtracting exciton photoinduced absorption, which is included in the SI. Plot $\mathrm{h}$ ) shows deconvoluted polaron kinetics (exciton contribution has been substracted). See Table S4 for details of the fits.

Now we discuss the decay dynamics of the photogenerated charges in the blends and devices. Only the composite blend shows clear intensity-dependent decays, which start at $\approx 10 \mathrm{ps,} \mathrm{and}$ exhibit a half time of $\sim 4$ ns at the highest intensity studied. This intensity dependent behaviour is 
also present in the total charge density on the ns timescale measured with TDCF, where a much faster decay is observed when the pulse intensity is increased by a factor of 10 . From the fits of these decays (not shown) a predominant second order kinetics can be obtained.

The scenario is completely different for the intercalated and non-intercalated systems. In the first case, a fast intensity-independent decay of the polaron absorption is observed. This decay can be fitted to a multi-exponential decay with an average time constant of $\tau=220 \pm 20$ ps (see Table S4). After $\sim 2$ ns $80 \%$ of the charges are lost to this fast charge recombination, in agreement with other studies. ${ }^{22,42,44,45}$ The TDCF data also show an intensity-independent firstorder recombination process, in agreement with ns-TAS measurements by Laquai et al. ${ }^{22}$ Our external quantum efficiency (EQE) measurements in the near IR (see Figure S7) show a band at $\sim 980 \mathrm{~nm}$, which suggests that there are bound species with an absorption coupled to the ground state that can generate free charges. These species therefore correspond to charge-transfer states. We envision that recombination of the most tightly bound states results in fast geminate recombination that starts from early times, leaving the loosely bound polaron pairs to survive recombination at longer time scales, and being more prone to be separated and extracted by a sufficiently large bias.

For the non-intercalated system, for which our simulations predict efficient free charge generation, the polaron TAS decays are surprisingly intensity-independent; however they undergo an exponential decay with a much larger time constant, $(\tau=1.43 \pm 0.08 \mathrm{~ns})$ compared with the fully intercalated system. Importantly, charge recombination follows a first order decay also in TDCF, while charge extraction is rather slow: notice that charges are extracted only after $\approx 100 \mathrm{~ns}$ ( see $n_{\text {pre }}$ in blue triangles in Figure S8e and S8f). 
Another important observation is the lack of near-IR absorption in our EQE measurements (see Figure S7) indicating that if there are bound species, their concentration is small and/or their coupling to the ground state is weak. Altogether, this evidences a scenario where charge pairs created at the donor:acceptor interface, after exciton dissociation, separate rapidly, thereby lowering their free energy, (see Figure 2) however only few of these pairs split further to become fully independent charge carriers able to reach the electrodes. This suggests that the charges might remain morphologically confined in small polymer or fullerene domains. Alternatively, given the broad density of states distribution and a low electron mobility of higher adduct fullerenes, electrons may become readily trapped preventing an efficient charge extraction in the device. ${ }^{37,47}$ Both scenarios are plausible, and it is likely that a combination of both is responsible for the low efficiencies of free charge formation and predominant first order recombination in this device, however the exact mechanism is beyond the scope of this contribution.

This study reveals important differences in the charge carrier dynamics of PBTTT:fullerene blends depending on the heterojunction morphology and domain purity. For the 1:1 PBTTT:PC ${ }_{70} \mathrm{BM}$ blend with an intercalated morphology, our simulations and TDCF data show free carrier formation to proceed through a (coulombically) bound precursor. However, as there are no internal domain boundaries, carriers can be efficiently extracted to the outside circuit with the assistance of an electric field. In agreement with this picture, geminate, first order polaron recombination dominates the kinetics in the absence of an electric field (as observed in TAS or TDCF near $V_{O C}$ ), but there is a noticeable increase of the EGE when reversing the bias, thus rendering a strong voltage-dependent photocurrent. These observations are consistent with a recent semi-empirical theoretical study of the effect of intermixed regions upon charge recombination. ${ }^{27}$ In contrast, in the non-intercalated 1:1 PBTTT:ICTA system, there is a shallow 
energetic barrier to separate the interfacial CT state, and yet most of the photogenerated charge carriers are either morphologically bound or energetically trapped charges in a more disordered blend. ${ }^{37}$ As a consequence, polaron recombination as obtained by TAS is first order, and the EGE remains at a low level independently of the applied voltage. An additional factor that can contribute to the poor charge separation and collection in the non-intercalated device may originate from the expected lower charge mobility of ICTA, as compared to $\mathrm{PC}_{70} \mathrm{BM},{ }^{48}$ which can hinder efficient charge generation and collection. ${ }^{11}$

It is only in the 1:4 PBTT:PC ${ }_{70} \mathrm{BM}$ system with a composite morphology, that the majority of photogenerated polarons fully separate, then exhibiting slow, second order recombination dynamics, and an overall higher EGE. We propose that in this system, charge separation benefits from the presence of larger and well-interconnected fullerene domains. It is also plausible that an energy cascade effect operates in the junction between the co-crystal and the semi-crystallized $\mathrm{PC}_{70} \mathrm{BM}$ areas with higher-electron-affinity. ${ }^{12,49}$ This is an effect unlikely to be present in the ICTA blends due to their small and non-crystallized domains, as can be observed in the WAXD data which does not show the characteristic peak at $q=1.37 \AA^{-1}$, proposed to come from aggregated fullerene areas. ${ }^{50,51}$ Note that this peak is only present for the composite blend where larger $\mathrm{PC}_{70} \mathrm{BM}$ domains are present. ${ }^{30,31}$

A second important conclusion comes from the direct comparison of TAS and TDCF data in Scheme 1 . Here, by assuming that the two measurements overlap at $\approx 6 \mathrm{ns,}$, and low excitation intensities $\left(2.2 \mu \mathrm{Jcm}^{-2}\right)$ we can obtain the polymer (hole) polaron extinction coefficient $\varepsilon_{P}$ from the TAS data, at the polaron maximum absorption. Using this approach, we estimate that $\varepsilon_{P}=(4.0 \pm 0.7) \times 10^{4} \mathrm{M}^{-1} \mathrm{~cm}^{-1}$ for PBTTT hole polarons, which is consistent with published data for other polymers used in OPV. ${ }^{52,53}$ We notice however, that this value was obtained using 
only the 1:1 and 1:4 PBTTT:PC ${ }_{70} \mathrm{BM}$ transients. Interestingly, for the non-intercalated system, the optical and optoelectronical methods do not seem to agree in the charge density obtained: the extractable charge density determined by TDCF is $\approx 2.6$ times lower than the total charge in the blend as estimated by TAS measurements. This is consistent with the idea that most of these charges are either morphologically or energetically trapped, rendering them vulnerable for geminate recombination and thus difficult to be extracted efficiently even under the influence of a strong bias. This is explicitly illustrated in the right panel of Scheme 1.
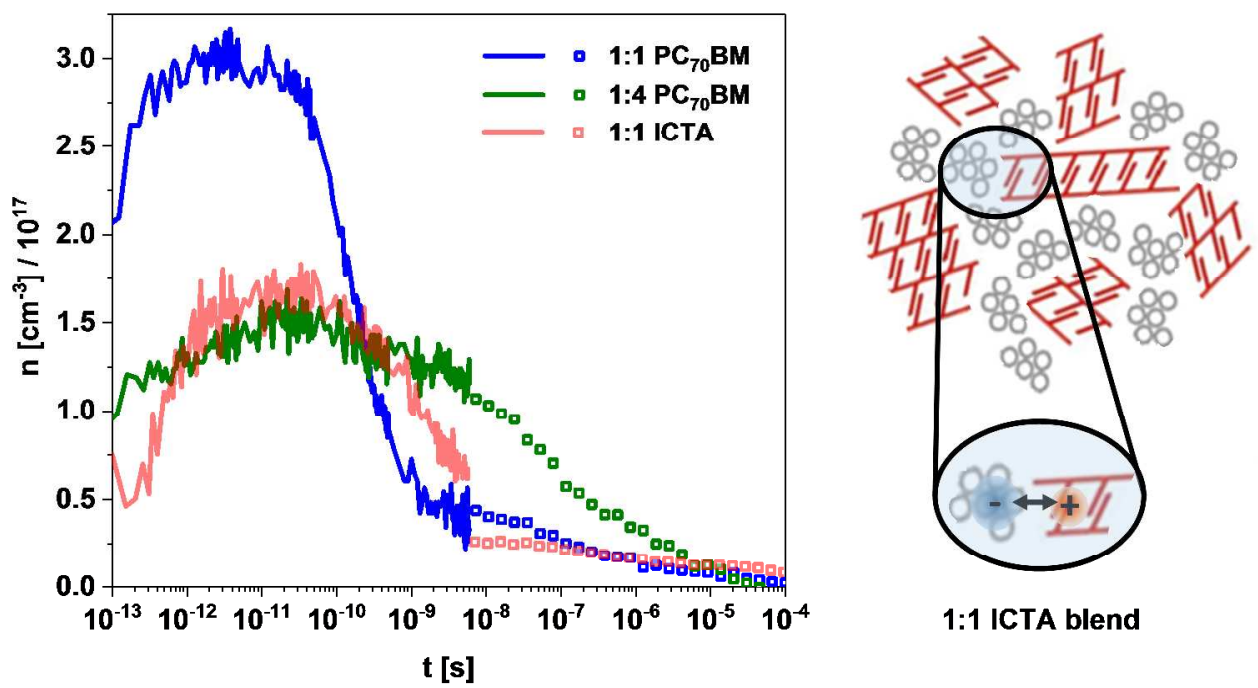

Scheme 1. Absolute polaron density kinetics from TAS (lines) on thin films and TDCF (open squares) on the corresponding devices for the three studied systems using pulse excitations at $540 \mathrm{~nm}$ and $2.2 \mu \mathrm{J} / \mathrm{cm}^{2}$. A hole polaron extinction coefficient of $(4.0 \pm 0.7) \times 10^{4} \mathrm{M}^{-1} \mathrm{~cm}^{-1}$ is obtained The depiction at the right illustrates the proposed morphology that is believed to be present in the 1:1 PBTTT:ICTA, non-intercalated blend and is responsible for the discrepancy observed from TAS and TDCF (see text).

Finally, it is worth noticing in Scheme 1 that "instantaneous" ( $\leq 200 \mathrm{fs})$ charge generation is actually more efficient in the $1: 1 \mathrm{PBTTT}: \mathrm{PC}_{70} \mathrm{BM}$ system, where in the range from $\approx 200$ fs to 45 ps polaron density is almost twice as high as for the other two systems. However, notice that charge generation as determined by TAS does not discriminate between bound or free charges; 
and after $\approx 200$ ps the charge density in the other two blends is higher due to their slower charge recombination. While it is important to understand the polaron pair kinetics from the ultrafast time scales, it is the free charge density at times relevant for charge extraction that is mostly relevant to device operation.

We have demonstrated the impact of the interface morphology upon charge generation, recombination and extraction in a combined kinetic study in blends and devices. While thermodynamic simulations show that charge separation is more efficient at an abrupt interface compared to an intercalated one, the former displays the lowest device efficiency. This is due to the inability of the non-intercalated system to form fully independent free charges in high mobility domains that favour efficient extraction. Remarkably, in both extreme cases - where either intercalated or phase-separated domains predominate - charges undergo geminate recombination of bound polaron pairs. In stark contrast, the composite 1:4 PBTTT:PC ${ }_{70} \mathrm{BM}$ system is the only one that results in good extraction and delayed, non-geminate recombination. This indicates that both intermixed and pure phases are required for an efficient device.

\section{METHODS.}

Film preparation. For optical measurements (fs-TAS, steady state PL) and device fabrication, solutions of PBTTT and $\mathrm{PC}_{70} \mathrm{BM}$ (or ICTA) in orto-dichlorobenzene (ODCB) were prepared at a concentration between $15-30 \mathrm{mg} \mathrm{mL}^{-1}$. The solutions were stirred and heated at $90^{\circ} \mathrm{C}$ for at least 8 hours to ensure full dissolution. The films were spun on cleaned glass substrates for 1 minute at $1500 \mathrm{rpm}$. For WAXD measurements, films were prepared by drop-casting $30 \mathrm{mg} \mathrm{mL}^{-}$ ${ }^{1}$ solutions of the neat polymer or the polymer/acceptor mixtures onto clean glass substrates. 
Wide-angle $X$-Ray diffraction (WAXD): the measurements were taken with a PANALYTICAL X'PERT-PRO Materials Research Diffractometer (MRD) equipped with a Nickel-filtered $\mathrm{Cu}$ $\mathrm{K} \alpha_{1}$ beam, with $\lambda=1.54 \AA$ and an $X^{\prime}$ CELERATOR detector. A current $\mathrm{I}=40 \mathrm{~mA}$ and an accelerating voltage $\mathrm{U}=40 \mathrm{kV}$ were used. Data was corrected to account for different film thicknesses and the baseline subtracted. Device fabrication. Pre-cleaned, patterned indium tin oxide (ITO) substrates (15 $\Omega$ per square) were used and treated with Oxygen plasma. On top of the ITO substrates, PEDOT:PSS was spun at $2500 \mathrm{rpm}$ and dried on a hot plate at $150^{\circ} \mathrm{C}$ in air for 30 minutes. Active layers for devices were prepared in a similar fashion as for the films used for optical measurements. Following, a counter electrode of calcium (10 nm) and aluminum (100 $\mathrm{nm}$ ) were deposited by vacuum evaporation at $3 \times 10^{-7}$ mbar. The active area of the devices used for TDCF was $0.01 \mathrm{~cm}^{2}$. Devices were encapsulated with blue fix glue.

Transient absorption spectroscopy (TAS): Measurements were carried out with a commercial setup that comprises a $1 \mathrm{kHz}$ Solstice (Newport Corporation) Ti:Sapphire regenerative amplifier with $800 \mathrm{~nm}, 90 \mathrm{fs}$ pulses. The output was passed through a beam splitter to generate the pump and probe pulses. The tuneable pump pulse was generated in a TOPAS-Prime (Light conversion) optical parametric amplifier and used to excite the sample with energies between 2 and $30 \mu \mathrm{Jcm}^{-2}$ at $540 \mathrm{~nm}$. The probe light was used to generate a Near-IR continuum (800-1400 nm) in a sapphire crystal. A HELIOS transient absorption spectrometer (Ultrafast Systems) was used for collecting transient absorption spectra and decays up to 6 ns. The time resolution of this set-up is 200 fs. The films were kept at all times under a Nitrogen atmosphere.

Time delayed collection field (TDCF). An optical pulse train generated by a diode-pumped, Qswitched Nd:YAG laser (NT242, EKSPLA, $500 \mathrm{~Hz}$ repetition rate, $3.8 \mathrm{~ns}$ pulse duration) was used to excite the sample. In the meantime, the device is held at a constant 'pre-bias' set by an 
Agilent 81150 A pulse generator trough a homebuilt amplifier and then switched to a strong reverse bias 'collection bias' after a certain delay time. The current through the device is measured via a grounded $10 \Omega$ resistor in series with the sample and recorded with an Agilent DSO9104H oscilloscope. To compensate for the internal latency of the pulse generator, the laser pulse was delayed and homogeneously scattered in an $85 \mathrm{~m}$ long silica fiber (LEONI).

\section{Supporting Information.}

Structures of the donor polymer and fullerene acceptors are included as well as UV-vis steadystate absorption of the respective neat and blend films. Details of the Thermodynamic simulations, optical spectroscopy (TAS and PL) complementary data. EQE and device efficiency figures and finally TDCF transient of devices are included. The Supporting Information is free of charge on the ACS Publication website.

\section{ACKNOWLEDGMENTS \\ This work was funded by UNVEiL, a BMBF project, the EPSRC (EP/IO1927B/1, EP/M023532/1 and EP/K011987/1) and the Welsh Assembly Government Sêr Cymru programme. ECF thanks CONACyT (scholarship 309929) and the Kernahan Fund from Imperial College London for funding. SNH and IK were supported by the Westpac Bicentennial Foundation and by the Australian Research Council through a Discovery Early Career Researcher Award (DE140100433) and through the Centre of Excellence for Engineered Quantum Systems (CE110001013).}




\section{REFERENCES}

(1) Gao, F.; Inganas, O. Charge Generation in Polymer-fullerene Bulk-Heterojunction Solar Cells. Phys. Chem. Chem. Phys. 2014, 16, 20291-20304.

(2) Few, S.; Frost, M.; Nelson, J. Models of Charge Pair Generation in Organic Solar Cells. Phys. Chem. Chem. Phys. 2014, 17, 2311-2325.

(3) Bässler, H.; Köhler, A. "Hot or Cold": How Do Charge Transfer States at the Donoracceptor Interface of an Organic Solar Cell Dissociate? Phys. Chem. Chem. Phys. 2015, $17,28451-28462$.

(4) Dimitrov, S. D.; Durrant, J. R. Materials Design Considerations for Charge Generation in Organic Solar Cells. Chem. Mater. 2013, 26, 616-630.

(5) Vandewal, K.; Albrecht, S.; Hoke, E. T.; Graham, K. R.; Widmer, J.; Douglas, J. D.; Schubert, M.; Mateker, W. R.; Bloking, J. T.; Burkhard, G. F. et al. Charge-Transfer States at Organic Interfaces. Nat. Mater. 2013, 13, 63-68.

(6) Bakulin, A. a; Rao, A.; Pavelyev, V. G.; van Loosdrecht, P. H. M.; Pshenichnikov, M. S.; Niedzialek, D.; Cornil, J.; Beljonne, D.; Friend, R. H. The Role of Driving Energy and Delocalized States for Charge Separation in Organic Semiconductors. Science, 2012, 335, 1340-1344.

(7) Savoie, B. M.; Rao, A.; Bakulin, A. A.; Gelinas, S.; Movaghar, B.; Friend, R. H.; Marks, T. J.; Ratner, M. A. Unequal Partnership: Asymmetric Roles of Polymeric Donor and Fullerene Acceptor in Generating Free Charge. J. Am. Chem. Soc. 2014, 136, 2876-2884.

(8) Niedzialek, D.; Duchemin, I.; de Queiroz, T. B.; Osella, S.; Rao, A.; Friend, R.; Blase, X.; Kümmel, S.; Beljonne, D. First Principles Calculations of Charge Transfer Excitations in Polymer-Fullerene Complexes: Influence of Excess Energy. Adv. Funct. Mater. 2015, 25, 1972-1984.

(9) Jones, M. L.; Dyer, R.; Clarke, N.; Groves, C. Are Hot Charge Transfer States the Primary Cause of Efficient Free-Charge Generation in Polymer:fullerene Organic Photovoltaic Devices? A Kinetic Monte Carlo Study. Phys. Chem. Chem. Phys. 2014, 16, 2031020320.

(10) Bernardo, B.; Cheyns, D.; Verreet, B.; Schaller, R. D.; Rand, B. P.; Giebink, N. C. Delocalization and Dielectric Screening of Charge Transfer States in Organic Photovoltaic Cells. Nat. Commun. 2014, 5, 3245.

(11) Philippa, B.; Stolterfoht, M.; Burn, P. L.; Juška, G.; Meredith, P.; White, R. D.; Pivrikas, A. The Impact of Hot Charge Carrier Mobility on Photocurrent Losses in Polymer-Based Solar Cells. Sci. Rep. 2014, 4, 5695.

(12) Jamieson, F. C.; Domingo, E. B.; McCarthy-Ward, T.; Heeney, M.; Stingelin, N.; Durrant, J. R. Fullerene Crystallisation as a Key Driver of Charge Separation in Polymer/fullerene Bulk Heterojunction Solar Cells. Chem. Sci. 2012, 3, 485. 
(13) Shoaee, S.; Subramaniyan, S.; Xin, H.; Keiderling, C.; Tuladhar, P. S.; Jamieson, F.; Jenekhe, S. A.; Durrant, J. R. Charge Photogeneration for a Series of Thiazolo-Thiazole Donor Polymers Blended with the Fullerene Electron Acceptors PCBM and ICBA. Adv. Funct. Mater. 2013, 23, 3286-3298.

(14) Ma, W.; Tumbleston, J. R.; Wang, M.; Gann, E.; Huang, F.; Ade, H. Domain Purity, Miscibility, and Molecular Orientation at Donor/acceptor Interfaces in High Performance Organic Solar Cells: Paths to Further Improvement. Adv. Energy Mater. 2013, 3, 864-872.

(15) Vandewal, K.; Himmelberger, S.; Salleo, A. Structural Factors That Affect the Performance of Organic Bulk Heterojunction Solar Cells. Macromolecules 2013, 46, 6379-6387.

(16) Mukherjee, S.; Proctor, C. M.; Bazan, G. C.; Nguyen, T.; Ade, H. Significance of Average Domain Purity and Mixed Domains on the Photovoltaic Performance of High-Effi Ciency Solution-Processed Small-Molecule BHJ Solar Cells. Adv. Energy Mater. 2015, 5, 1500877.

(17) Mukherjee Subhrangsu, X. J.; Ade, H. Charge Creation and Recombination in MultiLength Scale Polymer:Fullerene BHJ Solar Cell Morphologies. Adv. Energy Mater. 2016, $6,1-8$.

(18) Jiao, X.; Ye, L.; Ade, H. Quantitative Morphology-Performance Correlations in Organic Solar Cells: Insights from Soft X-Ray Scattering. Adv. Energy Mater. 2017, 1700084.

(19) Tumbleston, J. R.; Collins, B. a.; Yang, L.; Stuart, A. C.; Gann, E.; Ma, W.; You, W.; Ade, H. The Influence of Molecular Orientation on Organic Bulk Heterojunction Solar Cells. Nat. Photonics 2014, 8, 385-391.

(20) Ryno, S. M.; Fu, Y. T.; Risko, C.; Bredas, J. L. Polarization Energies at Organic-Organic Interfaces: Impact on the Charge Separation Barrier at Donor-Acceptor Interfaces in Organic Solar Cells. ACS Appl. Mater. Interfaces 2016, 8, 15524-15534.

(21) Kästner, C.; Egbe, D. A. M.; Hoppe, H. Polymer Aggregation Control in Polymerfullerene Bulk Heterojunctions Adapted from Solution. J. Mater. Chem. A 2015, 3, 395403.

(22) Gehrig, D. W.; Howard, I. A.; Sweetnam, S.; Burke, T. M.; Mcgehee, M. D.; Laquai, F. The Impact of Donor - Acceptor Phase Separation on the Charge Carrier Dynamics in pBTTT : PCBM Photovoltaic Blends. Macromol. Rapid Commun. 2015, 36, 1054-1060.

(23) Yan, H.; Song, Y.; McKeown, G. R.; Scholes, G. D.; Seferos, D. S. Adding Amorphous Content to Highly Crystalline Polymer Nanowire Solar Cells Increases Performance. Adv. Mater. 2015, 27, 3484-3491.

(24) Collado-Fregoso, E.; Deledalle, F.; Utzat, H.; Tuladhar, P. S.; Dimitrov, S. D.; Gillett, A.; Tan, C. H.; Zhang, W.; McCulloch, I.; Durrant, J. R. Photophysical Study of DPPTTT/PC70BM Blends and Solar Devices as a Function of Fullerene Loading: An Insight into EQE Limitations of DPP-Based Polymers. Adv. Funct. Mater. 2017, 27, 1-11. 
(25) Utzat, H.; Dimitrov, S. D.; Wheeler, S.; Collado-Fregoso, E.; Tuladhar, P. S.; Schroeder, B. C.; McCulloch, I.; Durrant, J. R. Charge Separation in Intermixed Polymer:PC70BM Photovoltaic Blends: Correlating Structural and Photophysical Length Scales as a Function of Blend Composition. J. Phys. Chem. C 2017, 121, 9790-9801.

(26) Lyons, B. P.; Clarke, N.; Groves, C. The Relative Importance of Domain Size, Domain Purity and Domain Interfaces to the Performance of Bulk-Heterojunction Organic Photovoltaics. Energy Environ. Sci. 2012, 5, 7657.

(27) Finck, B. Y.; Schwartz, B. J. Drift-Diffusion Studies of Compositional Morphology in Bulk Heterojunctions: The Role of the Mixed Phase in Photovoltaic Performance. Phys. Rev. Appl. 2016, 6, 1-16.

(28) Miller, N. C.; Sweetnam, S.; Hoke, E. T.; Gysel, R.; Miller, C. E.; Bartelt, J. A.; Xie, X.; Toney, M. F.; McGehee, M. D. Molecular Packing and Solar Cell Performance in Blends of Polymers with a Bisadduct Fullerene. Nano Lett. 2012, 12, 1566-1570.

(29) Miller, N. C.; Cho, E.; Gysel, R.; Risko, C.; Coropceanu, V.; Miller, C. E.; Sweetnam, S.; Sellinger, A.; Heeney, M.; McCulloch, I. et al. Factors Governing Intercalation of Fullerenes and Other Small Molecules between the Side Chains of Semiconducting Polymers Used in Solar Cells. Adv. Energy Mater. 2012, 2, 1208-1217.

(30) Miller, N. C.; Cho, E.; Junk, M. J. N.; Gysel, R.; Risko, C.; Kim, D.; Sweetnam, S.; Miller, C. E.; Richter, L. J.; Kline, R. J. et al. Use of X-Ray Diffraction, Molecular Simulations, and Spectroscopy to Determine the Molecular Packing in a PolymerFullerene Bimolecular Crystal. Adv. Mater. 2012, 24, 6071-6079.

(31) Buchaca-Domingo, E.; Ferguson, a. J.; Jamieson, F. C.; McCarthy-Ward, T.; Shoaee, S.; Tumbleston, J. R.; Reid, O. G.; Yu, L.; Madec, M.-B.; Pfannmöller, M. et al. AdditiveAssisted Supramolecular Manipulation of Polymer:fullerene Blend Phase Morphologies and Its Influence on Photophysical Processes. Mater. Horizons 2014, 1, 270.

(32) Wade, J.; Wood, S.; Collado-Fregoso, E.; Heeney, M.; Durrant, J. R.; Kim, J.-S. Impact of Fullerene Intercalation on Structural and Thermal Properties of Organic Photovoltaic Blends. J. Phys. Chem. C 2017, Submitted.

(33) Hood, S. N.; Kassal, I. Entropy and Disorder Enable Charge Separation in Organic Solar Cells. J. Phys. Chem. C 2016, 7, 4495-4500.

(34) Clarke, T. M.; Durrant, J. R. Charge Photogeneration in Organic Solar Cells. Chem. Rev. 2010, 110, 6736-6767.

(35) Gregg, B. A. Entropy of Charge Separation in Organic Photovoltaic Cells: The Benefit of Higher Dimensionality. J. Phys. Chem. Lett. 2011, 2, 3013-3015.

(36) Poelking, C.; Cho, E.; Malafeev, A.; Ivanov, V.; Kremer, K.; Risko, C.; Brédas, J. L.; Andrienko, D. Characterization of Charge-Carrier Transport in Semicrystalline Polymers: Electronic Couplings, Site Energies, and Charge-Carrier Dynamics in Poly(bithiopheneAlt-Thienothiophene) [PBTTT]. J. Phys. Chem. C 2013, 117, 1633-1640. 
(37) Nyman, M.; Sandberg, O. J.; Österbacka, R. Charge Transport in Intercalated and NonIntercalated Polymer:fullerene Blends. Synth. Met. 2015, 201, 6-10.

(38) Kniepert, J.; Schubert, M.; Blakesley, J.; Neher, D. Photogeneration and Recombination in P3HT: PCBM Solar Cells Probed by Time Delayed Collection Field Experiments. J. Phys. Chem. Lett. 2011, 700-705.

(39) Kurpiers, J.; Neher, D. Dispersive Non-Geminate Recombination in an Amorphous Polymer:Fullerene Blend. Sci. Rep. 2016, 6, 26832.

(40) Zusan, A.; Vandewal, K.; Allendorf, B.; Hansen, N. H.; Pfl, J.; Salleo, A.; Dyakonov, V.; Deibel, C. The Crucial Influence of Fullerene Phases on Photogeneration in Organic Bulk Heterojunction Solar Cells. Adv. Energy Mater. 2014, 4, 1400922.

(41) Rance, W. L.; Ferguson, A. J.; McCarthy-Ward, T.; Heeney, M.; Ginley, D. S.; Olson, D. C.; Rumbles, G.; Kopidakis, N. Photoinduced Carrier Generation and Decay Dynamics in Intercalated and Non-Intercalated Polymer:fullerene Bulk Heterojunctions. ACS Nano 2011, 5, 5635-5646.

(42) Scarongella, M.; Paraecattil, A. A.; Buchaca-Domingo, E.; Douglas, J. D.; Beaupré, S.; McCarthy-Ward, T.; Heeney, M.; Moser, J.-E.; Leclerc, M.; Fréchet, J. M. J. et al.The Influence of Microstructure on Charge Separation Dynamics in Organic Bulk Heterojunction Materials for Solar Cell Applications. J. Mater. Chem. A 2014, 2, 62186230 .

(43) Dou, F.; Buchaca-Domingo, E.; Sakowicz, M.; Rezasoltani, E.; McCarthy-Ward, T.; Heeney, M.; Zhang, X.; Stingelin, N.; Silva, C. The Effect of Phase Morphology on the Nature of Long-Lived Charges in Semiconductor Polymer:fullerene Systems. J. Mater. Chem. C 2015, 3, 3722-3729.

(44) Scarongella, M.; De Jonghe-Risse, J.; Buchaca-Domingo, E.; Causa, M.; Fei, Z.; Heeney, M.; Moser, J. E.; Stingelin, N.; Banerji, N. A Close Look at Charge Generation in Polymer: Fullerene Blends with Microstructure Control. J. Am. Chem. Soc. 2015, 137, 2908-2918.

(45) Causa, M.; De Jonghe-Risse, J.; Scarongella, M.; Brauer, J. C.; Buchaca-Domingo, E.; Moser, J.-E.; Stingelin, N.; Banerji, N. The Fate of Electron-hole Pairs in Polymer:fullerene Blends for Organic Photovoltaics. Nat. Commun. 2016, 7, 12556.

(46) Dimitrov, S.; Schroeder, B.; Nielsen, C.; Bronstein, H.; Fei, Z.; McCulloch, I.; Heeney, M.; Durrant, J. Singlet Exciton Lifetimes in Conjugated Polymer Films for Organic Solar Cells. Polymers,. 2016, 8, 14.

(47) Lenes, M.; Shelton, S. W.; Sieval, A. B.; Kronholm, D. F.; Hummelen, J. C.; Blom, P. W. M. Electron Trapping in Higher Adduct Fullerene-Based Solar Cells. Adv. Funct. Mater. 2009, 19, 3002-3007.

(48) Faist, M. A.; Shoaee, S.; Tuladhar, S.; Dibb, G. F. A.; Foster, S.; Gong, W.; Kirchartz, T.; Bradley, D. D. C.; Durrant, J. R.; Nelson, J. Understanding the Reduced Efficiencies of 
Organic Solar Cells Employing Fullerene Multiadducts as Acceptors. Adv. Energy Mater. 2013, 3, 744-752.

(49) Groves, C. Suppression of Geminate Charge Recombination in Organic Photovoltaic Devices with a Cascaded Energy Heterojunction. Energy Environ. Sci. 2013, 6, 1546.

(50) Piersimoni, F.; Chambon, S.; Vandewal, K.; Mens, R.; Boonen, T.; Gadisa, A.; Izquierdo, M.; Filippone, S.; Ruttens, B.; D’haen, J.; Martin, N.; Lutsen, L.; Vanderzande, D.; Adriaensens, P.; Manca, J. V. Influence of Fullerene Ordering on the Energy of the Charge-Transfer State and Open-Circuit Voltage in Polymer:fullerene Solar Cells. J. Phys. Chem. C 2011, 115, 10873-10880.

(51) Tumbleston, J. R.; Yang, L.; You, W.; Ade, H. Morphology Linked to Miscibility in Highly Amorphous Semi-Conducting Polymer/fullerene Blends. Polymer, 2014, 55, 4884-4889.

(52) Shuttle, C. G.; O’Regan, B.; Ballantyne, A. M.; Nelson, J.; Bradley, D. D. C.; Durrant, J. R. Bimolecular Recombination Losses in Polythiophene: Fullerene Solar Cells. Phys. Rev. $B, \mathbf{2 0 0 8}, 78,1-4$.

(53) Tautz, R.; Da Como, E.; Limmer, T.; Feldmann, J.; Egelhaaf, H.-J.; von Hauff, E.; Lemaur, V.; Beljonne, D.; Yilmaz, S.; Dumsch, I.; Allard, S.; Scherf, U. Structural Correlations in the Generation of Polaron Pairs in Low-Bandgap Polymers for Photovoltaics. Nat. Commun. 2012, 3, 970. 


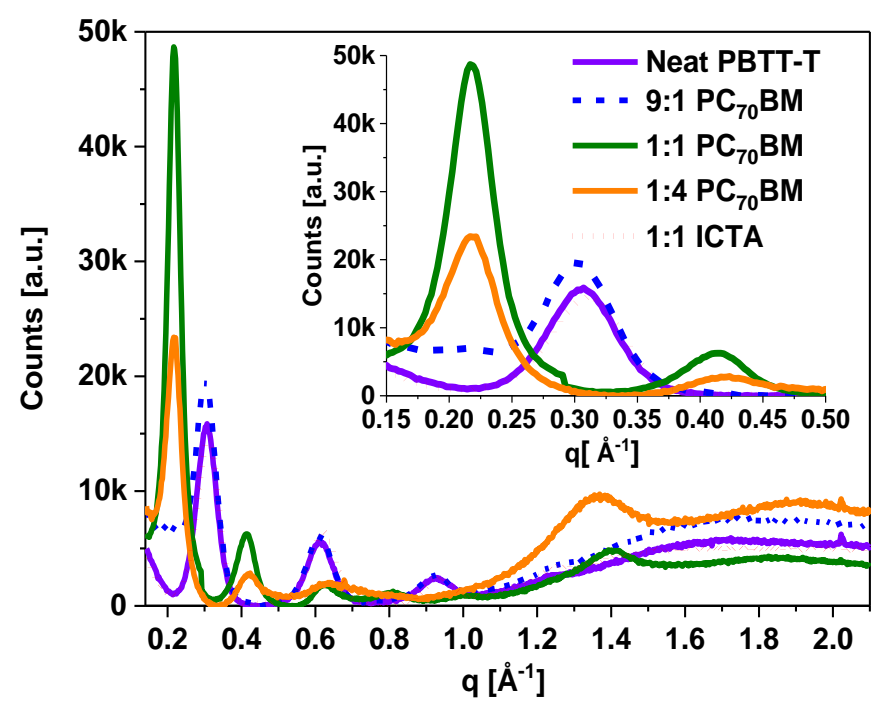

Figure 1. Wide-angle X-ray diffraction of neat PBTTT and PBTTT/fullerene thin films with different fullerene loadings. 


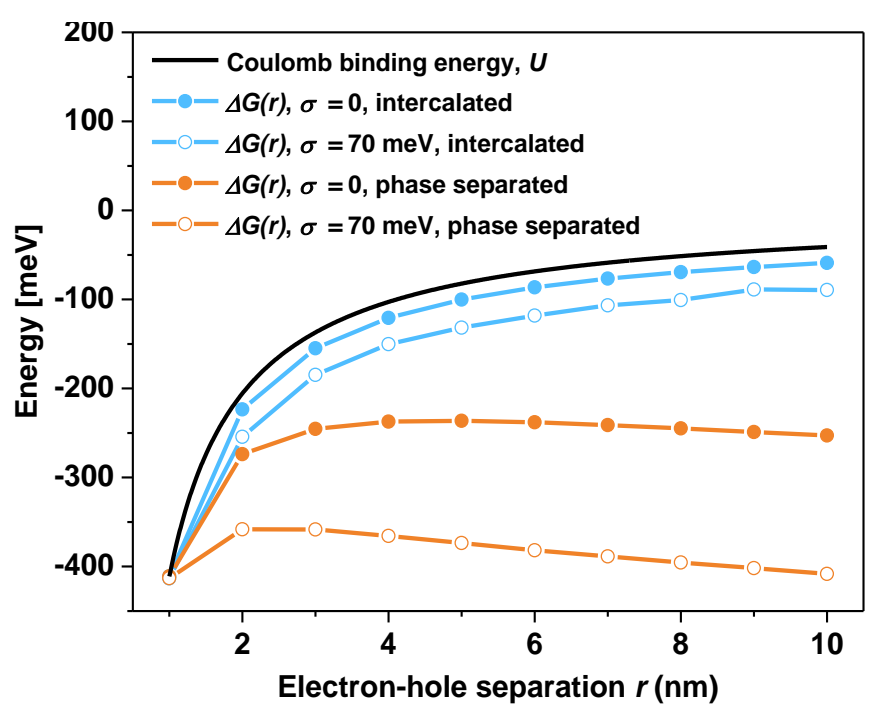

Figure 2. Electron-hole Coulombic binding (black curve) and free energy calculated without $(\sigma=0)$ and with $70 \mathrm{meV}$ of a Gaussian energetic disorder for a completely intercalated morphology (full circles) and for a phase separated system (empty circles). 


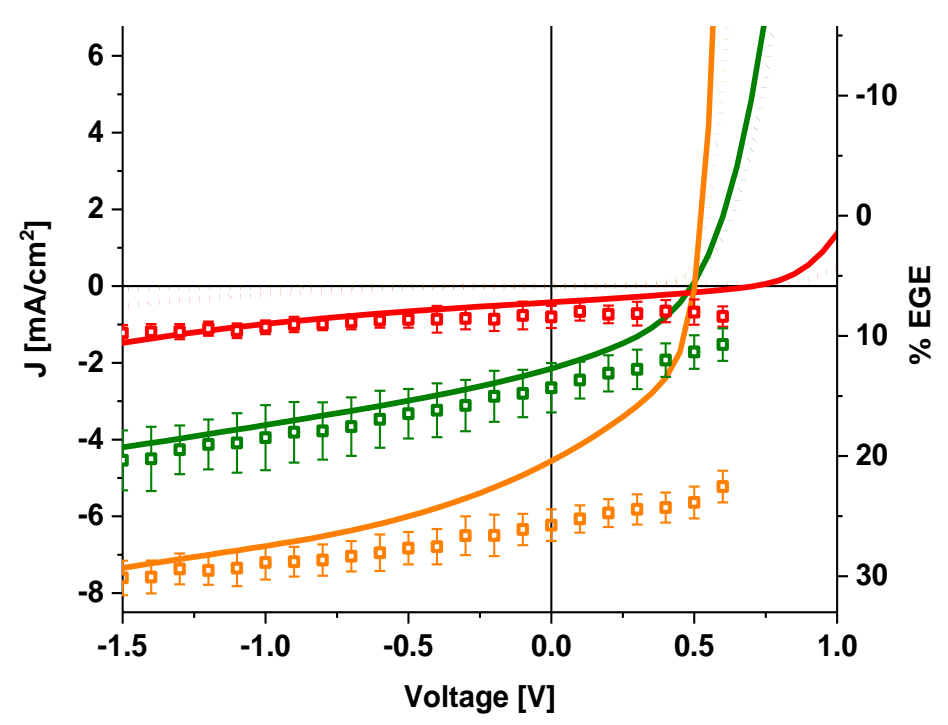

Figure 3. $J-V$ curves of representative, bulk heterojunction devices (ITO/PEDOT:PSS/active blend/Ca/Al) under constant, $100 \mathrm{mWcm}^{-2}$, AM 1.5 spectrum from a solar simulated light at room temperature. The active blends are composed of: (w/w) 1:4 PBTTT/PC ${ }_{70} \mathrm{BM}$ (orange curve), 1:1 PBTTT/PC $70 \mathrm{BM}$ (green curve) and 1:1 PBTTT/ICTA (red curve). Dotted lines correspond to the dark currents of the corresponding devices. Open squares represent the external generation efficiency as obtained by TDCF at $6 \mathrm{~ns}$ and using $-2.5 \mathrm{~V}$ for the collection bias after excitation at $540 \mathrm{~nm}$ and $0.2 \mu \mathrm{Jcm}^{2}$. (see text) 

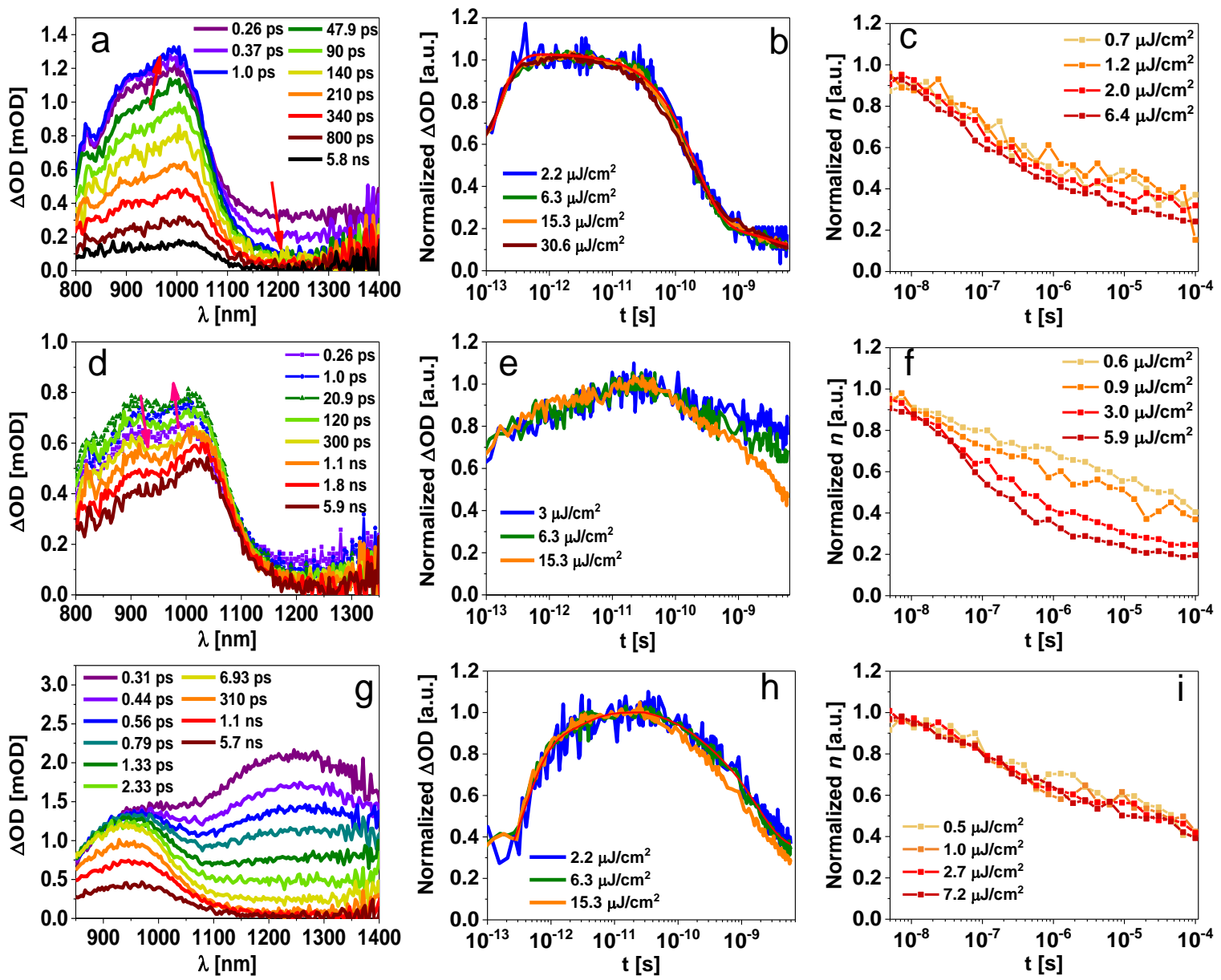

Figure 4. The three studied systems, one in each row: (a, b, c) 1:1 PBTTT:PC ${ }_{70} B M$ (intercalated), (d, e, f) 1:4 PBTTT:PC $70 \mathrm{BM}$ (composite) and (g, h, i) 1:1 PBTTT:ICTA (nonintercalated). Columns show: (a, d, g) plots of transient absorption (TAS) spectra exciting at $540 \mathrm{~nm}$ with $6 \mu \mathrm{J} / \mathrm{cm}^{2},(\mathrm{~b}, \mathrm{e}, \mathrm{h})$ normalized polaron recombination kinetics from TAS at the polaron absorption maximum, at different excitation intensities; and (c, f, i) normalized charge density from time-delayed collection field (TDCF) measurements at $0.4 \mathrm{~V}$ prebias and different light pulse intensities. Red lines in plot $b$ and $h$ correspond to tri-exponential fits of the data. Plot g) shows the raw TAS spectra before subtracting exciton photoinduced absorption, which is included in the SI. Plot h) shows deconvoluted polaron kinetics (exciton contribution has been substracted). See Table S4 for details of the fits. 

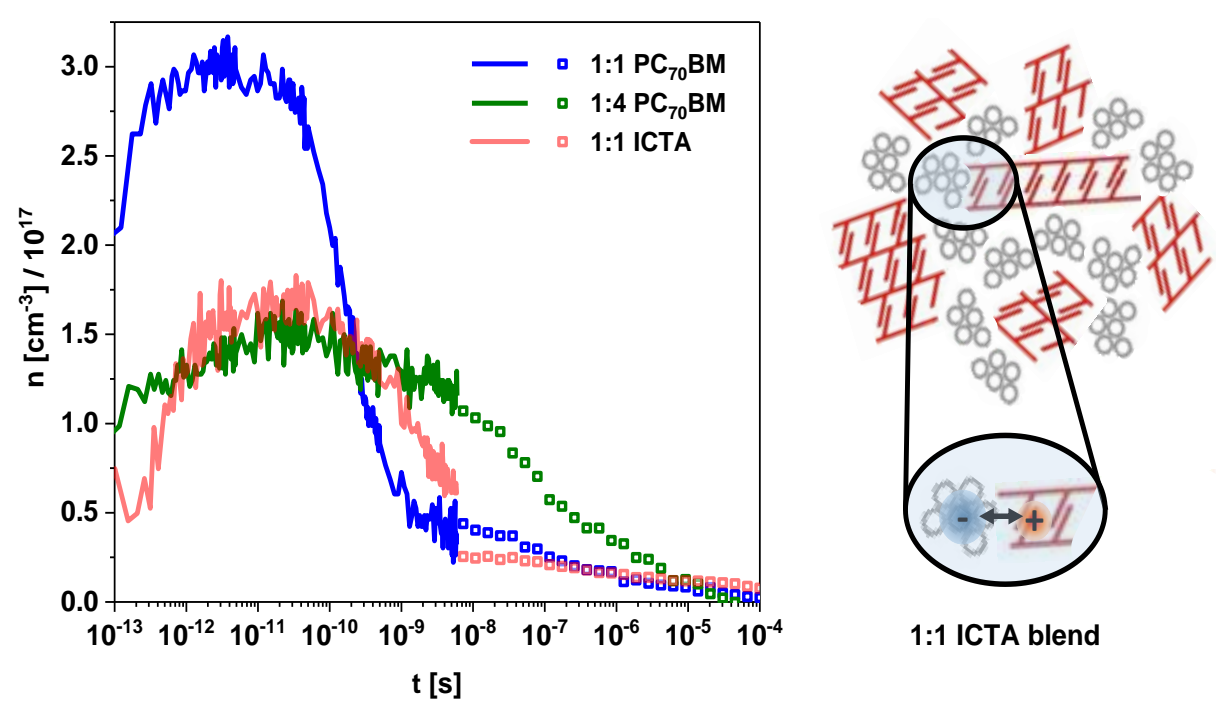

Scheme 1. Absolute charge density kinetics from TAS (lines) on thin films and TDCF (open squares) on the corresponding devices for the three studied systems using pulse excitations at $540 \mathrm{~nm}$ and $2.2 \mu \mathrm{J} / \mathrm{cm}^{2}$. A hole polaron extinction coefficient of $(4.0 \pm 0.7) \times 10^{4} \mathrm{M}^{-1} \mathrm{~cm}^{-1}$ is obtained The scheme at the right illustrates the proposed morphology that is believed to be present in the 1:1 PBTTT:ICTA blend and is responsible for the discrepancy observed from TAS and TDCF (see text). 